\title{
Optimization and research of primary frequency compensation strategy of the unit with supercritical forced circulation boiler
}

\author{
Ying-kun $\mathrm{Han}^{1}{ }^{1}{ }^{2}$, Lin $\mathrm{Mou}^{3}$, Wei-ming Liu², Peng-wei Yang ${ }^{2}$, Xiang-Kun Pang ${ }^{1}$ \\ ${ }^{1}$ State Grid Shandong Electric Power Research Institute, 250002 Jinan,Shandong, China \\ ${ }^{2}$ State Grid East Inner Mongolia Electric Power Research Institute, 010070Hohhot, Inner Mongolia, China \\ ${ }^{3}$ Shandong University Engineering Training Center, 250061 Jinan,Shandong , China
}

\begin{abstract}
The paper introduces the theory and application of Power Plant's primary frequency compensation(PFC) on the unit with the supercritical forced circulation boiler. First of all,the paper gives the typical parameters Setting and logic design of PFC. Then it explains some adverse factors which affect the PFC of the unit. With some problems in the operative process, the typical control strategy and some advices are given, which can improve PFC ability of Power Plant's PFC.
\end{abstract}

\section{Introduction}

With the development of new equipment and new technology, the power thermal units with supercritical forced circulation boiler will become the mainstream of technology due to the energy saving advantages. However, because of the technical characteristics on the reduction of heat storage capacity, the PFC capability of the units with the forced circulation boiler is weakened correspondingly. The PFC support to the power grid is affected. At the same time with the rapid development of the UHV grid and clean energy generation, more and more factors affect the power grid operation frequency. The large power grid safe and reliable operation with strong PFC ability is also more important. The units PFC ability has become an important technical support to the coordinated development on source-grid power system.

In order to improve the power quality and the control performance of the grid frequency in the operation, the coordination control system of the units must be optimized. The units are required to have the corresponding PFC capability. The paper will introduce the control principle and the typical technology solutions of PFC on the unit with the supercritical thermal unit. Combining the problems happened in the operative process, how to optimize PFC strategy can be illustrated. The correct control plan can avoid the adverse effect of PFC to the safe operation of the unit.

\section{The overview of primary frequency compensation(PFC)}

In the actual operation of the power grid, it is affected by the change of the user's consumption load, which causes the small and periodic changes of the power grid frequency. It is mainly based on the control system of the turbine generator to adjust the turbine valves automatically, which can complete the load compensation of the power grid and modify the fluctuation of the power grid frequency. This process is called PFC of the generator unit ${ }^{[1]}$.

The PFC function in DEH system, speed $\operatorname{deviation}(\Delta \mathrm{n}=$ rated speed-actual speed $)$ is usually used instead of frequency deviation $(\Delta \mathrm{f}=$ rated frequencyactual frequency), which is usually converted to power signal compensation or flow compensation. The control structure schematic diagram of PFC is shown in figure $1^{[2]}$.

For example, the power grid rated frequency is $50 \mathrm{~Hz}$ (the turbine rated speed is $3000 \mathrm{RPM}$ ). The PFC function (FG) converts $\Delta \mathrm{n}$ (rated speed and turbine actual speed difference) to a PFC compensation factor. If PFC ON, the PFC compensation factor will be added with the load reference value to control the load of the unit. If PFC OFF, the PFC factor is set to zero, that not to participate in the system control.

\footnotetext{
* Corresponding author: hanyk8086@163.com
} 


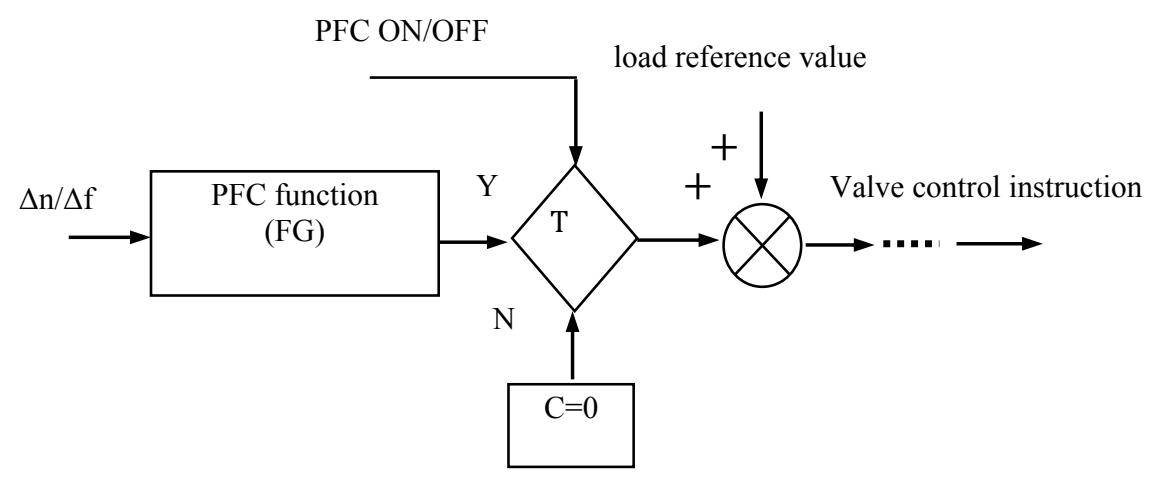

Figure 1 Control structure schematic diagram of PFC

\section{Typical parameters setting of PFC function (FG) ${ }^{[3]}$}

With the improvement of users' demand for power quality and frequency, the power grid has stringent requirements on PFC performance of grid-connected units. Therefore, the parameters setting of PFC is more important. The common PFC parameter setting methods are mainly used.

\subsection{No dead zone, no limit setting mode}

No dead zone, no limit setting mode. As long as the actual speed of the unit is deviated from the rated speed, the power grid frequency is adjusted. The control valves of the turbine are operated, which is unfavorable to the stable operation of the system. Limited to the capacity and stability performance requirements of China's grid, this setting mode is generally not applicable to the current generating sets.

\subsection{With dead zone, no limit setting mode}

With dead zone, no limit setting mode. The units can be set speed dead zone in this mode. When the $\Delta \mathrm{n}$ is in the range of the deviation dead zone, PFC OFF. When the $\Delta \mathrm{n}$ is beyond the deviation dead zone, PFC ON.

\subsection{With dead zone, no change $\delta$, no limit setting mode}

With dead zone, no change $\delta$ (speed governing droop), no limit setting mode. Compared with 3.2, this model can set the PFC dead zone. In the frequency difference of dead zone, the PFC factor has sudden change at the critical point, which will make the valves of the unit suddenly switch action. It will cause the changes of fuel, water supply, air supply and other parameters in the coordinated control system, which give rise to operation unstable of the system.

\subsection{With dead zone, with limit setting mode}

With dead zone, with limit setting mode. Compared with 3.3 , this setting mode to increase the PFC factor limit setting function can effectively prevent the frequency difference signal from overloading. The parameters can also be modified to realize one-way limit function, which can meet the technical requirements of the power grid.

\subsection{An instance of PFC function setting}

For example, the unit with supercritical forced circulation boiler that rated capacity is 600MW.It's PFC control method use DEH+CCS. According to the related technical requirements of power grid, speed governing droop is set to $5 \%$. At same time, speed dead zone is set to $\pm 2 \mathrm{RPM}$. The maximum compensation requirement of PFC is $6 \%$ of the rated load. The mode of the dead zone and limit setting is adopted in 3.4, and the set value of PFC command function is shown in table 1

Table 1 PFC function setting

\begin{tabular}{|c|c|c|}
\hline signs & $\begin{array}{c}\triangle \mathrm{n}(\mathrm{RPM}) \\
\mathrm{n}=\text { rated speed-actual speed }\end{array}$ & $\begin{array}{c}\text { Load } \\
\text { compensation } \\
\text { command } \\
(\mathrm{MW})\end{array}$ \\
\hline 2 & -11 & -36 \\
\hline 3 & -2 & 0 \\
\hline 4 & 2 & 0 \\
\hline 5 & 11 & 36 \\
\hline 6 & 152 & 36 \\
\hline
\end{tabular}
2.

The setting curve of PFC function is shown in figure

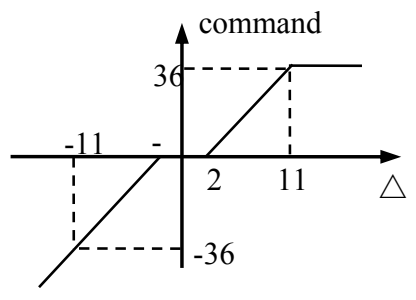

Figure 2 the setting curve of PFC function on 
When $|\triangle n|>2$, the controller will calculate the load compensation command according to the PFC function and $\triangle \mathrm{n}$, that add to the load reference value as the new command to control valve changing and complete unit's PFC function. When $|\triangle \mathrm{n}|<=2$, the compensation command is 0 , PFC OFF.

\section{Problems in the control logic of PFC}

In order to ensure the safety and reliability of the unit in actual operation, the fixed range is generally used to set the PFC. When the frequency modulation happens, this mode ensure the unit can not exceed the stable operation range. However, due to the improper logic configuration

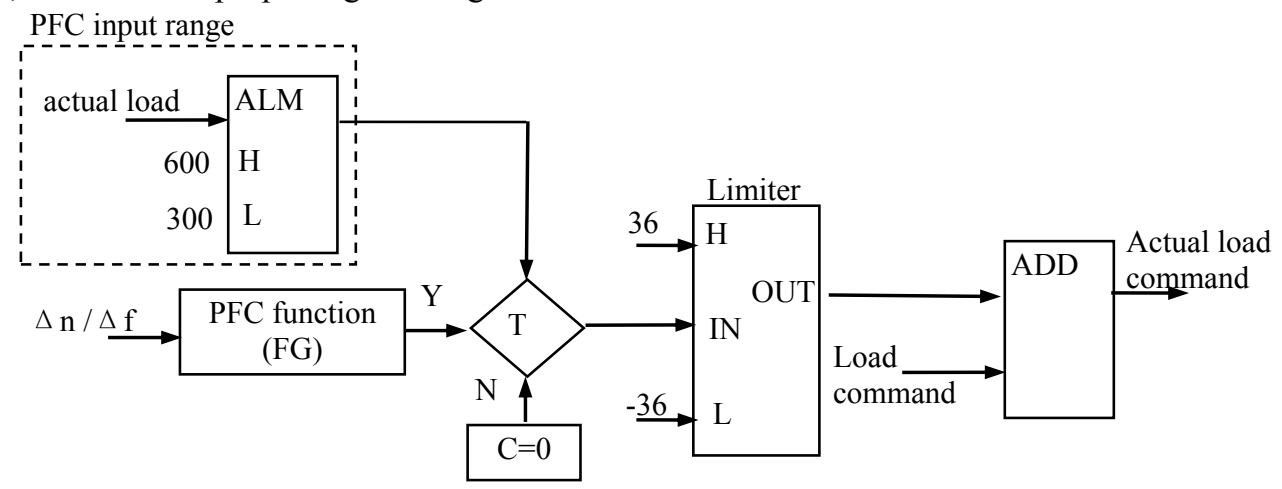

Figure 3 the diagram of improper input range on PFC

Figure 3 shows the logic configuration. When the unit's load is between $300 \mathrm{MW}$ and 600MW, the ALM module outputs is 1,PFC ON. When the unit's load is less than $300 \mathrm{MW}$ or the unit's load is greater than $600 \mathrm{MW}$, the ALM module outputs is 0, PFC OFF. The fixed input range in the virtual frame is guaranteed PFC exit automatically when the unit's load exceeds the range of $300 \mathrm{MW}-600 \mathrm{MW}$. But at limiting points, such as at the $303 \mathrm{MW}$ load point, PFC command is $-6 \mathrm{MW}$. When the unit's load is less than $300 \mathrm{MW}$, PFC command change to 0 , so actual load command will be increased. When the unit's load is greater than $300 \mathrm{MW}$, PFC IN again. The PFC command made load reduction and so on, that appear frequently $\mathrm{ON}$ or $\mathrm{OFF}$ on PFC. This configuration setting often causes the unit's load oscillate at the limit points, which is not conducive to the safe operation of the unit.

\subsection{The PFC compensation command with improper limit setting logic}

Figure 4 shows the logic configuration. A 600MW unit's configuration of PFC setting purpose is $36 \mathrm{MW}$ which is maximum value of PFC load compensation command. The unit's PFC range is $300 \mathrm{MW}$ to $600 \mathrm{MW}$. At the in the load range limitation, some units can not provide the correct limit protection, but also adversely affect the safe operation of the unit. Here are some improper logical configuration methods.

\subsection{Improper input conditions}

For example, a $600 \mathrm{MW}$ unit is set as shown in figure 3. The unit's PFC ideal range is $300 \mathrm{MW}-600 \mathrm{MW}$, and the PFC compensation load is set to $-36 \mathrm{MW}-+36 \mathrm{MW}$. The PFC will be OFF automatically when unit's load exceeds the stable operating range. limiting points, the controller will adjust PFC load compensation to the unit, which made operation load shall not exceed the limit value. Such as at the 590MW load point, if there is a PFC compensation command 20MW, the controller automatic computes the PFC command, that maximum is $10 \mathrm{MW}$, up to the highest command for maximum load range 600MW, not $610 \mathrm{MW}$. In order to ensure operation safety of the units, the load command can not exceed the maximum limit value.

Figure 4 the logic configuration calculates PFC compensation command, which select unit's actual load as comparing parameter. This mode will not affect the PFC adjustment under normal circumstances. When the load near the high or low load limit points, the PFC command may not meet the rating load, which affect the unit's PFC performance. For example, at the 590MW load point, the unit's command is 590MW before PFC action. The value will not change in PFC process. When PFC compensation command is $10 \mathrm{MW}$, the unit's actual load commands become 600MW. The unit rise load immediately. However in this process, the controller calculates the PFC compensation command gradually decreases. The unit's actual load command decreases. 


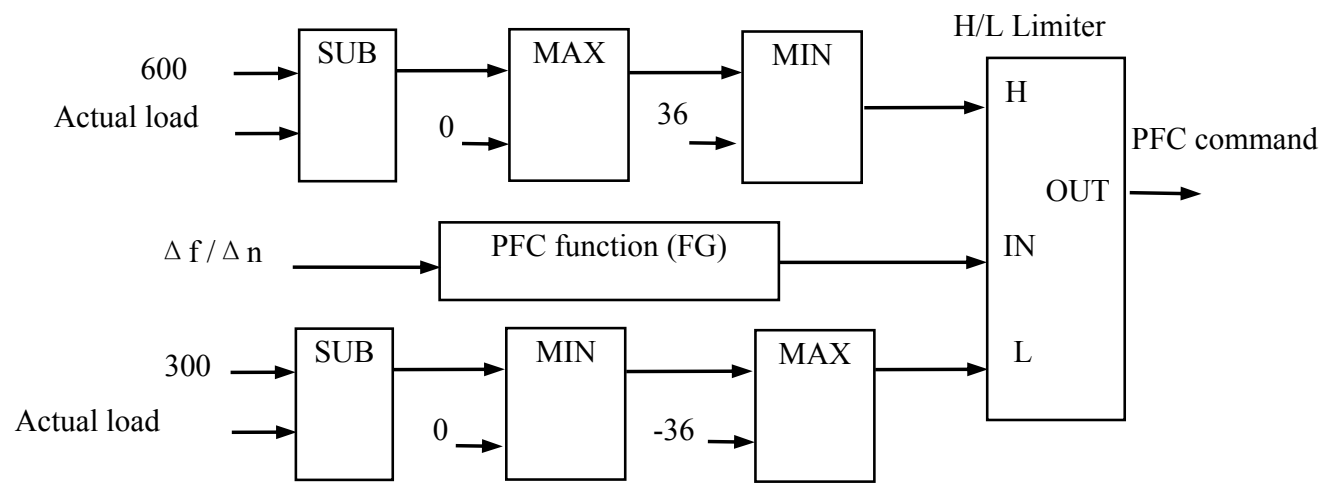

Figure 4 the diagram of PFC command logic

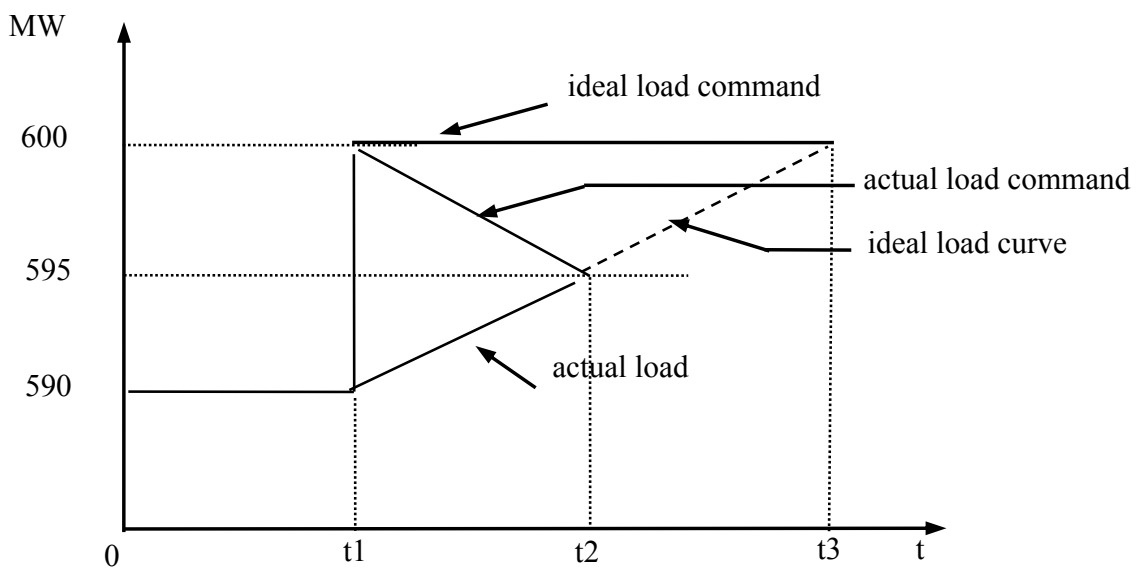

Figure 5 the response curve of PFC

The PFC process curve is shown in figure 5. If the PFC process is ideally, the unit's actual load command should equal to the ideal load command, that the actual load both are $600 \mathrm{MW}$ finally, at the time point $\mathrm{t} 3$. Before time point $\mathrm{t} 1$, the load command and the actual load curve is overlapping, the values both are 590MW. The unit is operating stable. At time point t1, the PFC compensation load command is up to load 10MW because PFC happen,. The PFC compensation load command add with load command become the ideal load command as $600 \mathrm{MW}$. From t1 to t2, PFC is operating. The load increase and PFC commands automatically reduce at the same time, due to the actual load command decreases. Finally the unit load stability in time point $\mathrm{t} 2$, at this time the unit's actual load command and the unit's load both are 595MW, which do not meet the technical index of $\mathrm{PFC}$ requirements.

\section{Adverse factors affect the PFC}

\subsection{Low parameters operation}

Considering the running efficiency, most of the unit adopts variable parameters at present, that is in different load, the parameters in different pressure. The units keep the turbine valves can be higher opening degree, in order to reduce the throttle of the adjustment and improve the economy. However, the units are operating at the higher opening degree, which has great impact on the PFC of the units. When the valves opening exceeds a certain value, its flow characteristic is poor. For example,the unit's PFC on, if increase load command, load compensation may be limited because the flow characteristic is poor. This mode will affect the unit's PFC capability.

\subsection{Turbine following mode}

When the unit is operating under the turbine following mode (TF mode), the control object of the turbine is the main steam pressure. When the frequency is adjusted, the load of the units is modulated according to the fluctuation of the power grid frequency. At the same time, the main steam pressure may be changed and modulated. When the controller modulates the main pressure in the TF mode, the process of the PFC is counterproductive. If the grid frequency is less than the rated frequency, the unit's PFC compensation load will rise, which make the main steam pressure drop. In order to adjust the main steam pressure, valves will be down, which will make the unit load drop. When the unit is running in $\mathrm{TF}$ mode that without special pressure 
correction scheme, the units PFC performance obviously can't meet the design requirements.

\subsection{Control valves with poor linearity}

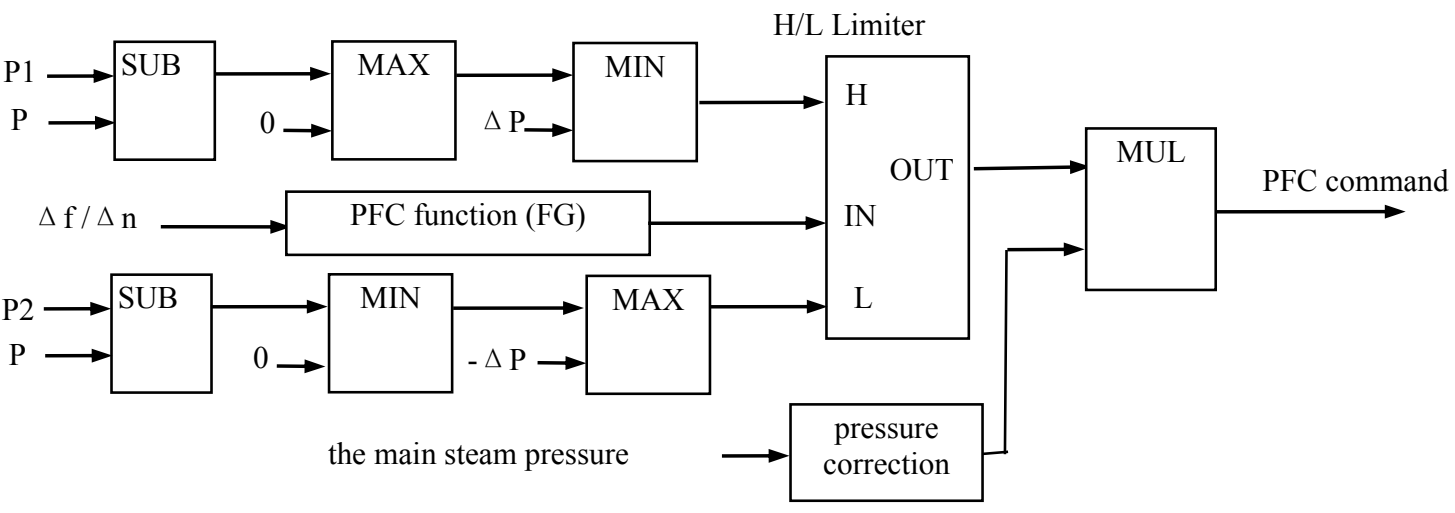

Figure 5 the diagram of an optimal design scheme

Notes: P: instantaneous power load command (no including PFC command)

P1: PFC working upper limit

P2: PFC working lower limit

$\Delta \mathrm{f} / \Delta \mathrm{n}$ : frequency fluctuation difference/ the turbine speed fluctuation

$\Delta \mathrm{P}: \quad \mathrm{PFC}$ ideal load command

\section{An optimal design scheme for the PFC}

The control logic configuration is shown in figure 6 . Based on instantaneous power load command P, PFC upper limit P1 and lower limit P2, the controller automatically calculates the limiting values to set $\mathrm{H} / \mathrm{L}$ limiter module on high limit value and low limit value. According to the grid frequency fluctuation difference $\Delta \mathrm{f}$ (usually use the turbine speed fluctuation $\Delta \mathrm{n}$ instead of $\Delta \mathrm{f}$ ) and PFC function (FG), the controller can calculate out the ideal PFC load command. Then the PFC ideal load command use $\mathrm{H} / \mathrm{L}$ limiter module to guarantee system working in the normal adjustment range. The PFC compensation command is in range of $\Delta \mathrm{P} \sim \Delta \mathrm{P}$, which can prevent a large disturbance and guarantee the unit's safe and stable.

According to the real-time main steam pressure, the controller calculates a PFC correction coefficient by the pressure correction function block, which will adjust the PFC command. The correct PFC command can ensure the unit's PFC action under various operating mode. The unit's PFC can meet the design requirements, at same time it can ensure the quality of power supply.

\section{Conclusion}

As power system requirements to improve the performance of power-grid coordination, the power unit's PFC are usually using in the mode with DEH+CCS. IN DEH system, the PFC has a quickly response speed, that can be operated easily, and don't have big impact on
When the control valves had been operating for long time, especially the unit valves after maintenance, the flow curve will drifted. If the false flow curve is set to the control valves, the control performance of the unit will get worse. The effect to PFC load compensation is not reliable at the same time.

$\mathrm{H} / \mathrm{L}$ Limiter

the stability of the unit. IN the coordinated control system (CCS), the PFC adjust the load accurately. Using in the mode with DEH+CCS, the unit's PFC ability can be guaranteed. The units with the correct logic configuration of PFC not only can meet the requirements of the PFC performance, but also can meet the needs of the unit stable operating.

\section{References}

1. Xiao Z. DEH of the Steam Turbine [M]. Beijing: China Electric Power Press, 2005.

2. Wang F. Automatic Control and Protection on Power Units [M]. Beijing: China Electric Power Press, 2005.

3. Han Y, Meng X, Mou L. Application and Research on 1000MW Power Plant's Control Mode of Frequency adjustment in DEH[J]. East China Electric Power,2008,Vol36,No3:95-97 